Research Article

\title{
Interaction of Agaric Acid with the Adenine Nucleotide Translocase Induces Mitochondrial Oxidative Stress
}

\author{
Edmundo Chávez, ${ }^{1}$ Mabel Buelna-Chontal $\mathbb{D}^{2},{ }^{2}$ Arturo Macías-López, ${ }^{2}$ \\ Luz Hernández-Esquivel, ${ }^{1}$ Francisco Correa $\mathbb{D}^{2},{ }^{2}$ and Natalia Pavón ${ }^{3}{ }^{3}$ \\ ${ }^{1}$ Departamento de Bioquímica, Instituto Nacional de Cardiología, Ignacio Chávez Ciudad de México, Mexico \\ ${ }^{2}$ Departamento de Biomedicina Cardiovascular, Instituto Nacional de Cardiología, Ignacio Chávez Ciudad de México, Mexico \\ ${ }^{3}$ Departamento de Farmacología, Instituto Nacional de Cardiología, Ignacio Chávez Ciudad de México, Mexico
}

Correspondence should be addressed to Francisco Correa; glicoproteico@hotmail.com and Natalia Pavón; pavitonat@ yahoo.com.mx

Received 20 February 2020; Accepted 5 December 2020; Published 22 December 2020

Academic Editor: Andrei Surguchov

Copyright (c) 2020 Edmundo Chávez et al. This is an open access article distributed under the Creative Commons Attribution License, which permits unrestricted use, distribution, and reproduction in any medium, provided the original work is properly cited.

\begin{abstract}
Mitochondrial permeability transition is characterized by the opening of a transmembranal pore that switches membrane permeability from specific to nonspecific. This structure allows the free traffic of ions, metabolites, and water across the mitochondrial inner membrane. The opening of the permeability transition pore is triggered by oxidative stress along with calcium overload. In this work, we explored if oxidative stress is a consequence, rather than an effector of the pore opening, by evaluating the interaction of agaric acid with the adenine nucleotide translocase, a structural component of the permeability transition pore. We found that agaric acid induces transition pore opening, increases the generation of oxygen-derived reactive species, augments the oxidation of unsaturated fatty acids in the membrane, and promotes the detachment of cytochrome c from the inner membrane. The effect of agaric acid was inhibited by the antioxidant tamoxifen in association with decreased binding of the thiol reagent eosin-3 maleimide to the adenine nucleotide translocase. We conclude that agaric acid promotes the opening of the pore, increasing ROS production that exerts oxidative modification of critical thiols in the adenine nucleotide translocase.
\end{abstract}

\section{Introduction}

An essential mitochondrial function is to provide the cell with the energetic requirements for its metabolic processes. To accomplish this, a selectively permeable membrane is required to guarantee the establishment of a proton gradient across the inner membrane. The nonselective permeability process, widely known as mitochondrial permeability transition (mPT), disrupts the proton gradient that powers oxidative phosphorylation [1]. The nonspecific pore has a diameter of up to $3 \mathrm{~nm}$ and allows the free traffic of ions, metabolites, and even DNA fragments [2]. The molecular nature of this nonspecific pore has been the matter of debate for the last 30 years and is still under debate. Early experiments pointed to adenine nucleotide translocase (ANT) $[3,4]$ or to the phosphate carrier $[5,6]$ as the main structure confirming the mitochondrial permeability transition pore (mPTP). Recently, the interphase between $c$ subunits of the $\mathrm{F}_{1}$-ATP synthase dimers has been pointed out as the nonspecific pore, and in this new model, ANT plays a regulatory role [7]. The pore is opened by a large number of inducers, including carboxyatractyloside [8], $\mathrm{Ca}^{2+}$ [9], thiol-blocking reagents, heavy metals [10-13], and oxidative stress. It is known that oxidative stress is a main factor that promotes mPTP opening in an isolated mitochondria $[9,14]$, in intact cells [15] and during reperfusion damage [16]. However, it is not clear if reactive oxygen species (ROS) generation is a consequence of pore opening, or if endogenously generated ROS accounts for its aperture. Thus, the objective of this work was to explore the possibility that the opening of the nonspecific pore may induce oxidative stress. We used agaric acid $(\mathrm{Ag} A \mathrm{c})$ to induce the aperture of the pore, as we 
previously reported its interaction with ANT [17]. The experimental results show that Ag Ac caused pore opening, resulting in drop of the transmembrane electric gradient $(\Delta \Psi)$. The collapse of $\Delta \Psi$ amplified $\mathrm{H}_{2} \mathrm{O}_{2}$ production, increased the oxidation of the lipid milieu of the inner membrane, and inhibited the activity of superoxide dismutase. ROS increase might result from detachment of cytochrome $\mathrm{c}$ from the inner membrane. According to these results, it is provocative to assume that the opening of the nonspecific pore promoted oxidative stress, as such an event appears to be inhibited by the oxidant molecule tamoxifen (TAM).

\section{Materials and Methods}

Chemicals were of reagent or higher grade from SigmaAldrich (St Louis, MO) unless otherwise specified. Anticytochrome c rabbit monoclonal antibody (Ab76237) was from Abcam (Cambridge, MA, USA), and anti-VDAC mouse monoclonal was (sc390996) from Santa Cruz Biotechnology (CA, USA). The enhanced chemiluminescence detection system was from Millipore Corporation (Bedford, MA, USA), and horseradish peroxidase- (HRP-) conjugated secondary antibodies were from Abcam (Cambridge, MA, USA).

The investigation was approved by the Ethics Committee of the National Institute of Cardiology, "Ignacio Chávez" (INC-13806), and the experimental protocols followed the guidelines of Norma Oficial Mexicana for the use and care of laboratory animals (NOM-062-ZOO-1999) and for disposal of biological residues (NOM-087-SEMARNAT-SSA1-2002).

2.1. Mitochondrial Protein. The mitochondria from the rat kidney cortex were prepared by homogenization of the tissue in $0.25 \mathrm{M}$ sucrose $1 \mathrm{mM}$ EDTA, $10 \mathrm{mM}$ TRIS adjusted with Tris to $\mathrm{pH} 7.3$, following standard centrifugation procedures. Protein content was determined following the method of Lowry et al. [18].

2.2. $\mathrm{Ca}^{2+}$ Retention Capacity, Mitochondrial Swelling, and Transmembrane Gradient Measure. The experiments were carried out by incubating $2 \mathrm{mg}$ of mitochondrial protein in $3 \mathrm{ml}$ of basic medium containing $125 \mathrm{mM} \mathrm{KCl}, 10 \mathrm{mM}$ succinate, $3 \mathrm{mM}$ phosphate, $10 \mathrm{mM}$ HEPES, $5 \mu \mathrm{g}$ rotenone, and $2 \mu \mathrm{g}$ oligomycin. The medium was adjusted to $\mathrm{pH} 7.3$. The movements of $\mathrm{Ca}^{2+}$ were assayed spectrophotometrically at $675-685 \mathrm{~nm}$ using the metallochromic indicator Arsenazo III. Mitochondrial swelling was followed at $540 \mathrm{~nm}$. The transmembrane electric gradient $(\Delta \Psi)$ was determined using the dye Safranine.

2.3. Superoxide Dismutase (SOD) Activity. The activity of the enzyme superoxide dismutase was analyzed in the mitochondria by nondenaturating acrylamide gel electrophoresis as described by Pérez-Torres et al. [19]. Briefly, it used 100 micrograms of mitochondrial protein in a nondenaturing gel electrophoresis and nitro blue tetrazolium
(NBT). The electrophoresis was carried out at $100 \mathrm{v}$ for $5 \mathrm{~h}$. At the end, the gel was incubated in $2.45 \mathrm{mM} \mathrm{NBT}$ for $30 \mathrm{~min}$, the liquid was discarded, and the gel was incubated in a $28 \mathrm{mM}$ TEMED solution containing $36 \mathrm{mM}$ potassium phosphate $(\mathrm{pH} \mathrm{7.8)}$ and $0,028 \mathrm{mM}$ riboflavin in the dark for $10 \mathrm{~min}$. The blue NBT stain was developed by exposure to UV light for another $10 \mathrm{~min}$. A standard curve was obtained with a serial dilution $(2.5,5,10,15,30$, and $60 \mathrm{ng})$ of SOD from bovine erythrocytes.

2.4. Lipid Peroxidation and $\mathrm{H}_{2} \mathrm{O}_{2}$ Content. Lipid peroxidation of the mitochondrial membrane was carried out with the thiobarbituric acid reactive substances using tetraethoxypropane as standard as previously described [13]. $\mathrm{H}_{2} \mathrm{O}_{2}$ content was evaluated according to the procedure of Dikalov et al. [20], by incubating $2 \mathrm{mg}$ of mitochondrial protein in $3 \mathrm{ml}$ of a basic medium and $10 \mu \mathrm{mol} \mathrm{L}^{-1} 10$ acetyl3,2-dihydrophenoxazine plus $0.2 \mathrm{U} / \mathrm{ml}$ horseradish peroxidase in a dark chamber at $37^{\circ} \mathrm{C}$ for $60 \mathrm{~min}$. The product, resorufin, was determined based on an increase in fluorescence at $530-590 \mathrm{~nm}$.

2.5. Mitochondrial DNA and Cytochrome C Content. Mitochondrial DNA was obtained as reported previously [2]; its disruption was resolved in $0.8 \%$ agarose gels and visualized with ethidium bromide under UV light.

2.6. The Content of Mitochondrial Cytochrome C. The content of cytochrome $\mathrm{C}$ was analyzed by loading $50 \mu \mathrm{g}$ of protein from the mitochondria onto 15\% SDS-PAGE gel and transferred to PVDF membranes for immunodetection. A primary monoclonal antibody against cytochrome $\mathrm{C}$ and a secondary HRP-conjugated antibody were used to evaluate the protein content of the membrane. The content of the voltage-dependent anion channel (VDAC) was used as loading control.

2.7. Adenine Nucleotide Translocase Detection. Adenine nucleotide translocase was labeled as previously reported by Majima et al. [21]; $1 \mathrm{mg}$ of mitochondrial protein was suspended in $1 \mathrm{ml}$ of a basic medium and preincubated with $3 \mu \mathrm{M}$ agaric acid and $20 \mu \mathrm{M}$ tamoxifen for $5 \mathrm{~min}$; subsequently, $20 \mathrm{nmol} / \mathrm{mg}$ eosin maleimide (EMA) was added and incubated for $5 \mathrm{~min}$ at $4^{\circ} \mathrm{C}$ in darkness. The reaction was stopped by adding $30 \mathrm{mM}$ DTT(dithiothreitol). Then, $300 \mu \mathrm{g}$ of mitochondrial protein was then subjected to SDS-PAGE in $10 \%$ polyacrylamide under nonreducing conditions. Fluorescence was detected with a UV-lamp.

\section{Results}

Figure 1(a)) shows the inhibitory effect of tamoxifen on the efflux of mitochondrial $\mathrm{Ca}^{2+}$ as induced by Ag Ac. Calcium was accumulated into mitochondria oxidizing succinate and reached a steady state until the addition of $3 \mu \mathrm{M}$ Ag Ac that initiated a fast release of this cation (I). This was completely abolished by cyclosporin A (CSA) (II), indicating the 


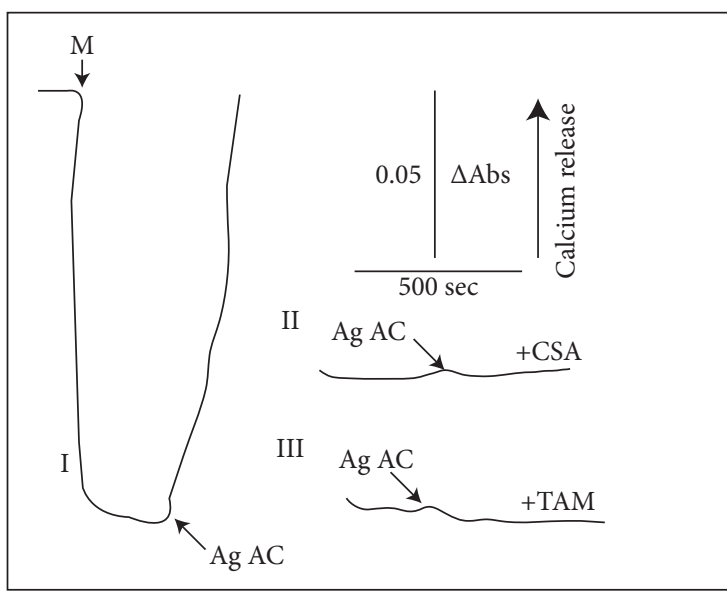

(a)

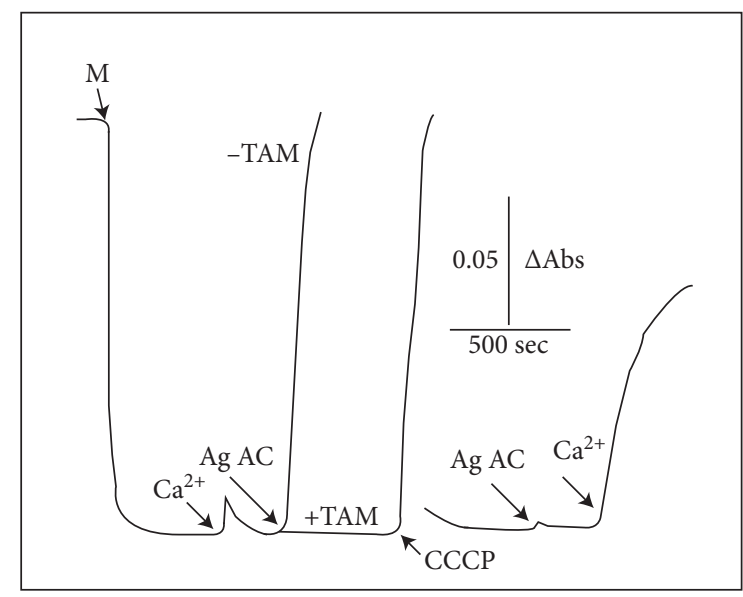

(b)

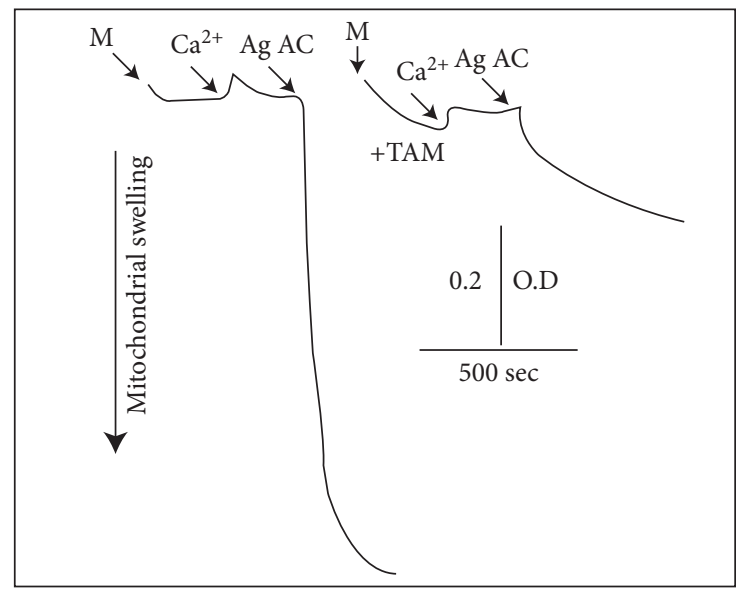

(c)

Figure 1: (a) Agaric acid effect on the matrix $\mathrm{Ca}^{2+}$ content release. Mitochondrial protein $(2 \mathrm{mg})$ was added to $3 \mathrm{ml}$ of a basic medium as described in Materials and Methods. Where indicated, $3 \mu \mathrm{M}$ agaric acid (Ag Ac), $2 \mu \mathrm{M}$ cyclosporin A (CSA), and $20 \mu \mathrm{M}$ tamoxifen (TAM) were added. Traces are representative of, at least, 4 different experiments. (b) Agaric acid-induced collapse of the transmembrane electric gradient. Mitochondrial protein was incubated in $3 \mathrm{ml}$ of a medium. Where indicated, $50 \mu \mathrm{M} \mathrm{CaCl}_{2}, 3 \mu \mathrm{M}$ agaric acid, $20 \mu \mathrm{M}$ tamoxifen, and $1 \mu \mathrm{M}$ of the uncoupler CCCP were added. (c) Attenuation by tamoxifen of the agaric acid-induced mitochondrial swelling. Mitochondrial protein was incubated in $3 \mathrm{ml}$ of basic. Where indicated, $50 \mu \mathrm{M} \mathrm{CaCl}_{2}, 3 \mu \mathrm{M}$ agaric acid, and $20 \mu \mathrm{M}$ tamoxifen were added. Temperature: $25^{\circ} \mathrm{C}$. Traces are representative of, at least, 4 different experiments.

mediation of the permeability transition pore. In (III), it is shown that $20 \mu \mathrm{M}$ of the antioxidant tamoxifen (TAM) also inhibited matrix $\mathrm{Ca}^{2+}$ efflux induced by Ag Ac.

Building up of the transmembrane electric gradient is an essential prerequisite to sustain oxidative phosphorylation. In Figure $1(\mathrm{~b})$, it is shown that $\mathrm{Ca}^{2+}$ exerts temporal depolarization that is corrected when the rates of uptake and release reach the equilibrium. The addition of $3 \mu \mathrm{M} \mathrm{Ag} \mathrm{Ac}$ exerted a fast drop of membrane potential; however, this feature of the permeability transition was eliminated when the medium was supplemented with TAM. It is also shown that the uncoupler carbonyl cyanide m-chlorophenylhydrazone (CCCP) dissipates the transmembrane electric gradient and that that $\mathrm{Ca}^{2+}$ is required to promote the collapse of $\Delta \Psi$ induced by Ag Ac (right panel).

Mitochondrial swelling also characterizes the permeability transition pore opening. The experimental results presented in Figure 1(c)) show that Ag Ac induced a fast and extensive swelling, which was inhibited after the addition of TAM.

Next, we explore the possibility that Ag Ac induces oxidative stress by measuring the generation of hydrogen peroxide. Ag Ac did augment the production of $\mathrm{H}_{2} \mathrm{O}_{2}$ (Figure 2(a)), and such rise appears to be inhibited after the addition of TAM.

Mitochondrial damage resulting from increased $\mathrm{H}_{2} \mathrm{O}_{2}$ levels would normally be ameliorated via the activity of the first line member of the mitochondrial antioxidant system, i.e., superoxide dismutase enzyme (SOD). As observed in Figure 2(b)), Ag Ac inhibited SOD activity by 50\%, and such inhibition was partially reversed in Tam-treated mitochondria.

Increased oxidative stress, resulting from augmented hydrogen peroxide levels and diminished SOD activity, promoted damage to lipids, DNA, and proteins in the mitochondria. The oxidation of membrane polyunsaturated fatty acids was 


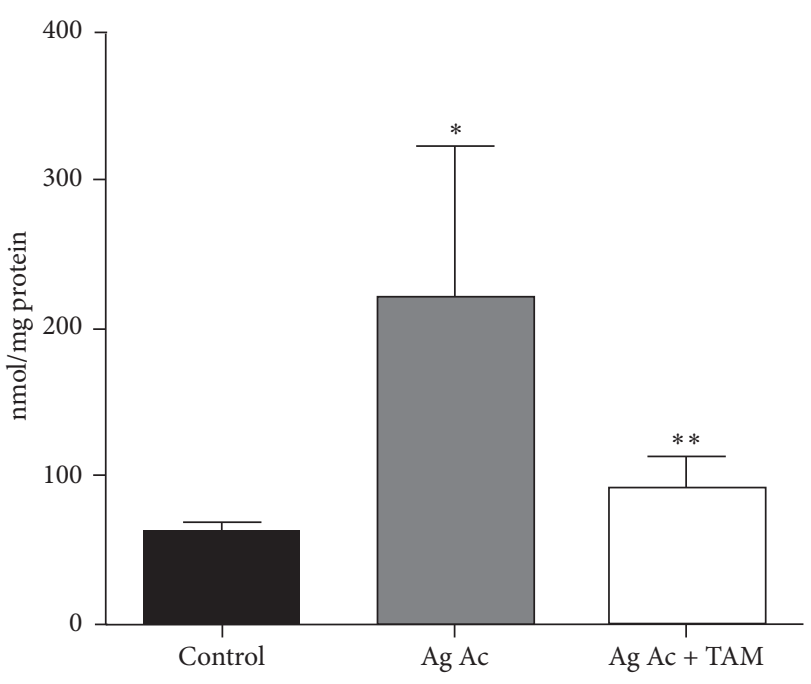

(a)

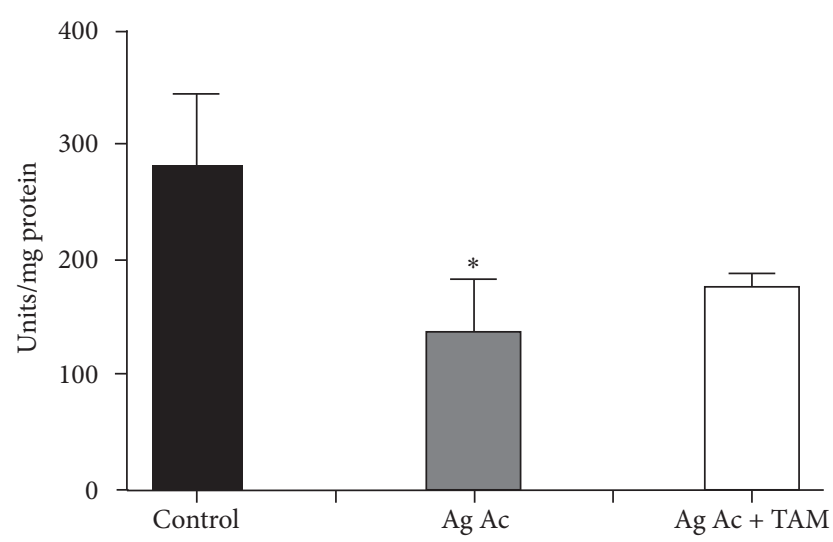

(b)

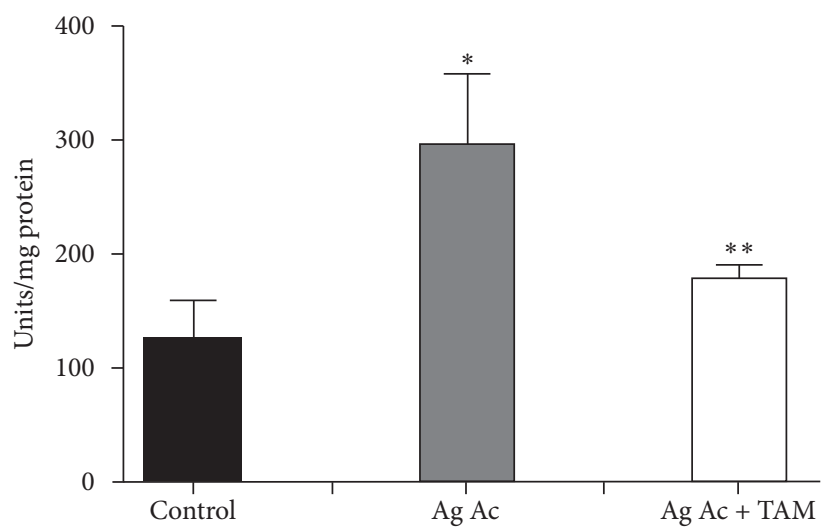

(c)

FIGURE 2: (a). Increased generation of hydrogen peroxide by agaric acid and its inhibition by tamoxifen. Mitochondrial protein ( $2 \mathrm{mg}$ ) was incubated in $3 \mathrm{ml}$ of a basic medium. Where indicated, $3 \mu \mathrm{M}$ agaric acid (Ag Ac) and $20 \mu \mathrm{M}$ tamoxifen were added. Results are the mean \pm SD of 3 different experiments. ${ }^{*} p<0.05$ vs. control; ${ }^{* *} p<0.05$ vs. Ag Ac. (b). Protective effect of tamoxifen on the agaric acidinduced inhibition of the superoxide dismutase enzyme. The added concentrations of agaric acid (Ag Ac) and tamoxifen were $3 \mu \mathrm{M}$ and $20 \mu \mathrm{M}$, respectively. The data represent the mean \pm SD of six different experiments. ${ }^{*} p<0.05$ vs. control; ${ }^{* *} p<0.05$ vs. Ag Ac. (c). Agaric acid-induced oxidation of the membrane lipid milieu and the inhibitory effect of tamoxifen. Mitochondrial protein ( $2 \mathrm{mg})$ was added to $3 \mathrm{ml}$ of a basic medium. Where indicated, $3 \mu \mathrm{M}$ agaric acid (Ag Ac) and $20 \mu \mathrm{M}$ tamoxifen (TAM) were added. The data represent the average of six different experiments $\pm \mathrm{SD}$ of six different experiments. ${ }^{*} p<0.05$ vs. control; ${ }^{* *} p<0.05$ vs. Ag Ac. Incubation temperature: $25^{\circ} \mathrm{C}$.

evaluated measuring the levels of thiobarbituric acid reactive substances (TBARS), which are produced as byproducts of lipid peroxidation. As observed, Tam diminished the oxidation of membrane fatty acids induced by Ag Ac (Figure 2(c)). Oxidative stress also induces damage on mitochondrial DNA (mtDNA). Figure 3 shows that Ag Ac induces the rupture of the genetic material. As is illustrated, mtDNA degradation was prevented by the antioxidant TAM.

It is known that mitochondrial permeability transition might contribute to apoptosis by releasing cytochrome $\mathrm{c}$ from the mitochondria $[22,23]$. In this context, we found that Ag Ac promoted the detachment of this protein from the inner membrane. As can be seen from Figure 4, cytochrome c content diminished by $40 \%$ in $\mathrm{Ag}$ Ac-treated mitochondria, and this decrement was partially abolished by TAM.
To determine if oxidative stress induced by Ag-Ac impacted on ANT, we labeled the mitochondria with the fluorescent probe eosin-5-maleimide (EMA). According to Majima et al. [21], this compound binds to Cys159 on the adenine nucleotide translocase, which has a molecular weight of $30 \mathrm{kDA}$. It is shown in Figure 5 that $\mathrm{Ag} \mathrm{Ac}$ reduced the fluorescent labeling of mitochondrial proteins by EMA, in particular of proteins weighting $30 \mathrm{kDa}$ (lines 4-6) in comparison with control mitochondria (lines 1-3). The addition of tamoxifen restored the fluorescent mark (lines 7-9), indicating either that tamoxifen prevents the binding of Ag Ac near the Cys159 locus or that by diminishing ROS production, it prevented the oxidative modification of critical thiols in the adenine nucleotide translocase. 


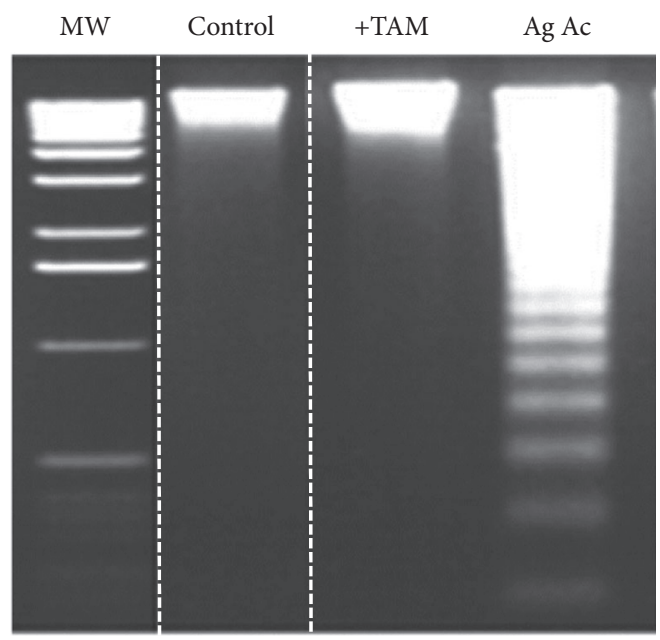

FIGURE 3: Effect of agaric acid on mitochondrial DNA disruption and its protection by the antioxidant tamoxifen. Experimental conditions were as described under Materials and Methods. Where indicated, $3 \mu \mathrm{M}$ agaric acid and $20 \mu \mathrm{M}$ tamoxifen (+Tam) were added. The image is representative of 3 different experiments.
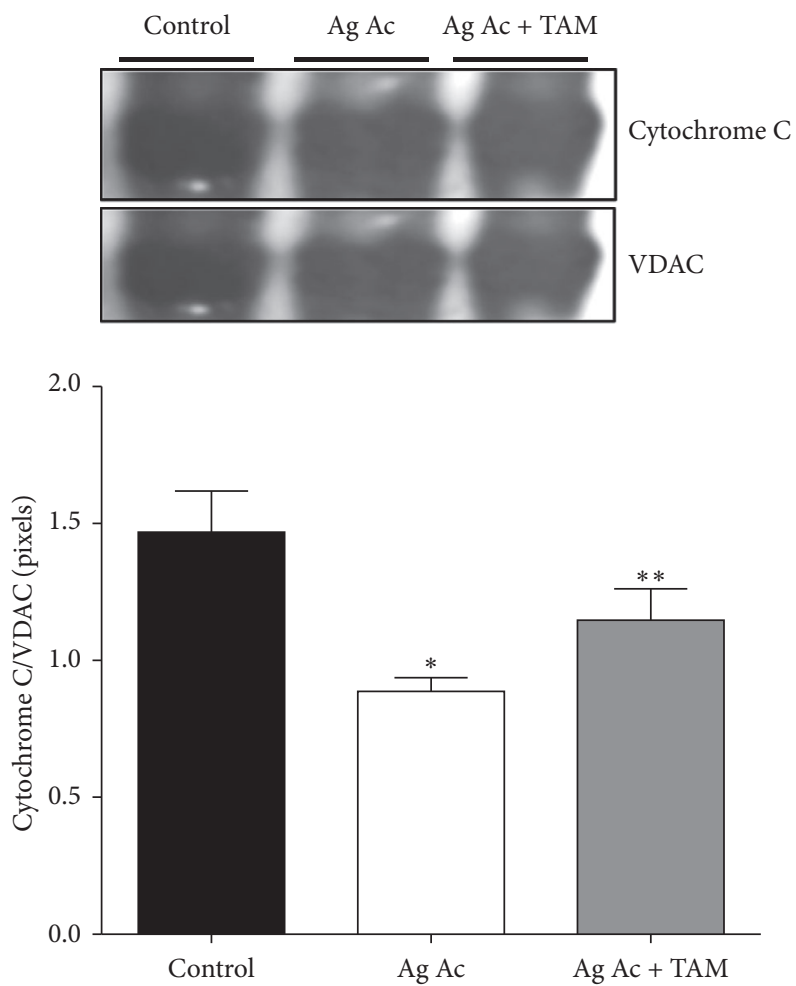

FIGURE 4: Agaric acid-induced detachment of cytochrome $\mathrm{c}$ and its protection by tamoxifen. Experimental conditions were as described in Materials and Methods. Data represent mean \pm SD of, at least, three different experiments ${ }^{*} p<0.05$ vs. control; ${ }^{* *} p<0.05$ vs. Ag Ac. The image is representative of, at least, 3 different experiments.

\section{Discussion}

Membrane pore permeability is an important biological phenomenon playing an essential role in normal biological processes [24], and its dysregulation turned out to be a crucial step in the pathogenesis of a variety of diverse diseases, encompassing ischemia-reperfusion damage [25], liver damage [26], many chronic and acute disorders of the central nervous system [27], and collagen VI myopathies [28].

Reports pointed out to ANT as a structural component of the nonspecific pore $[3,4]$. More recently, it has been proposed that the $c$ subunit of the F1-ATP synthase is the main mPTP constituent and that ANT plays a regulatory role in its 


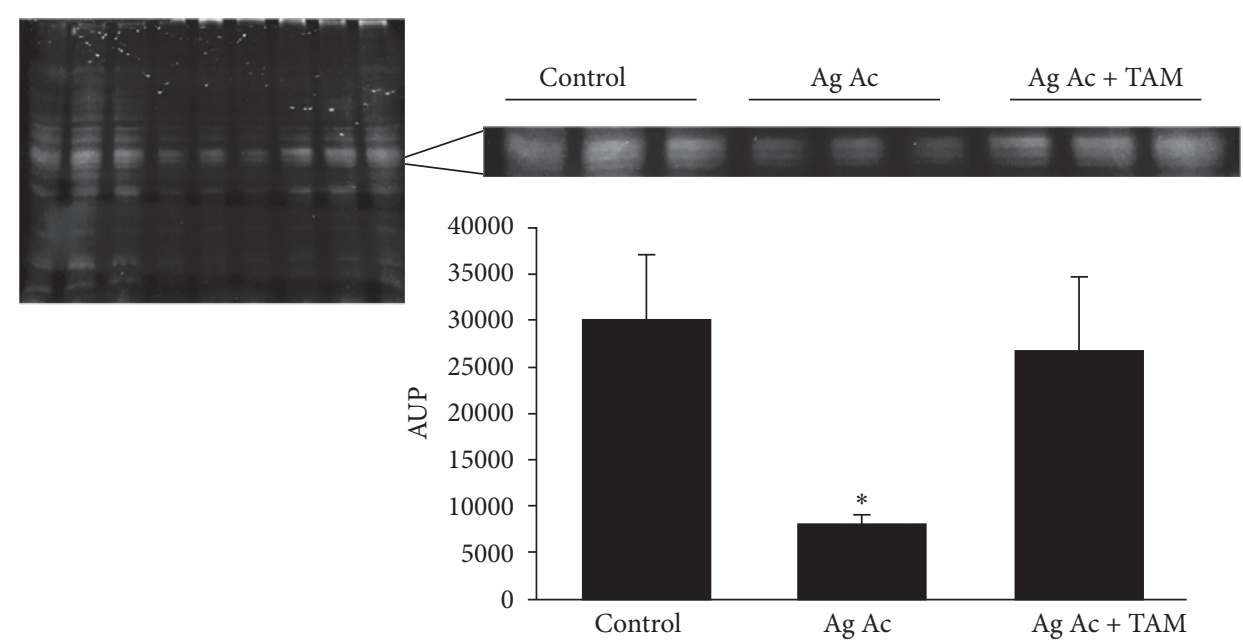

FIGURE 5: Effect of tamoxifen on the diminution of EMA binding to the adenine nucleotide translocase. Experimental conditions were as indicated in Materials and Methods. Where indicated, $3 \mu \mathrm{M}$ agaric acid (Ag Ac) and $20 \mu \mathrm{M}$ tamoxifen (TAM) were added.

open-closed state [7]. The pore opening is triggered under different experimental settings, i.e., by adding thiol blocking reagents [10] and heavy metals [11-13, 29] or by inhibiting the mitochondrial respiratory chain $[30,31]$, and also in physiopathological conditions such as ischemia/reperfusion damage [16]. Oxidative stress and mPTP opening are "hallmark" of the injury developed during reperfusion $[9,32,33]$. As antioxidants prevent from the aperture of the mPTP, it is accepted that the sudden generation of ROS derives in the aperture of the nonspecific pore. Contrary to such view, recent reports have claimed that the opening of the nonspecific pore is the cause of oxidative stress [34]. In this work, we add evidence to this possibility by using Ag Ac to induce the aperture of the nonspecific pore and Tam, a molecule with antioxidant properties [35]. ROS generation took place after the addition of Ag Ac, along with diminished SOD activity, oxidation of membrane unsaturated fatty acids, and release of cytochrome $c$ from the mitochondria. All these events were inhibited by the oxidant TAM.

There are reports showing that cytochrome $\mathrm{c}$ is released either before or after PT pore opening depending on the stimuli [36]. In the first scenario, cytochrome $c$ release would increase ROS generation after complex III inhibition [9] promoting PT pore opening, which, in turn, might lead to more cytochrome $c$ release and to further increase of ROS levels in a self-amplifying loop [37]. Another possible mechanism by which oxidative stress might be produced after the opening of a nonspecific pore is the close relationship between the transmembrane electric gradient and the generation of ROS [38]. Our experiments pointed out to this hypothesis, as agaric acid induced both a drop of $\Delta \Psi$, as well as the generation of $\mathrm{H}_{2} \mathrm{O}_{2}$.

Regarding the role of ANT in mPTP opening, we previously reported that Ag Ac might interact with the adenine nucleotide translocase [17] and also that Tam inhibits permeability transition as induced by carboxyatractyloside [39]. In this sense, an additional result that deserves special attention is that Tam prevented from the Ag Ac-induced decrease in EMA binding to ANT that might account for the protective effect of the antioxidant. It is known that Cys159 is the target site of EMA on adenine nucleotide translocase [21].

\section{Conclusions}

We propose that Ag Ac might interact with ANT through the cationic environment of a loop formed by Lys145, Arg151, Lys162, Lys165, and Arg170. This interaction might be reinforced through the interaction of the alkyl chain of $\mathrm{Ag}$ Ac with the hydrophobic milieu of the inner membrane. The hypothesis that such interaction induces pore opening is sustained by a previous publication [17], as well as by the results of Figure 1 in this work. Therefore, Tam reduces oxidative stress, either by preventing the binding of Ag Ac near the Cys159 locus or by acting as an antioxidant and preventing the oxidative modification of critical thiols in the adenine nucleotide translocase.

\section{Data Availability}

Data used to support the findings of this study are given in the manuscript.

\section{Conflicts of Interest}

The authors declare that they have no conflicts of interest.

\section{Acknowledgments}

The authors thank Dr. Cecilia Zazueta for her critical review of this manuscript.

\section{References}

[1] N. Mnatsakanian, G. Beutner, K. M. Alavian, and E. A. Jonas, "Physiological roles of the mitochondrial permeability transition pore," Journal of Bioenergetics and Biomembranes, vol. 49, pp. 13-25, 2017. 
[2] N. García and E. Chávez, "Mitochondrial DNA fragments released through the permeability transition pore correspond to specific gene size," Life Sciences, vol. 81, pp. 213-222, 2007.

[3] D. Macedo, M. d. F. Nepomuceno, and L. Pereira-da-Silva, "Involvement of the ADP/ATP carrier in permeabilization processes of the inner mitochondrial membrane," European Journal of Biochemistry, vol. 215, no. 3, pp. 595-600, 1993.

[4] A. Rück, M. Dolder, T. Wallimann, and D. Brdiczka, "Reconstituted adenine nucleotide translocase forms a channel for small molecules comparable to the mitochondrial permeability transition pore," FEBS Letters, vol. 426, no. 1, pp. 97-101, 1998.

[5] A. W. C. Leung, P. Varanyuwatana, and A. P. Halestrap, "The mitochondrial phosphate carrier interacts with cyclophilin D and may play a key role in the permeability transition," Journal of Biological Chemistry, vol. 283, no. 39, pp. 2631226323, 2008.

[6] P. Varanyuwatna and A. P. Halestrap, "The roles of phosphate and the phosphate carrier in the mitochondrial permeability transition pore," Mitochondrion, vol. 12, pp. 120-125, 2012.

[7] M. Bonora, A. Bononi, E. De Marchi et al., "Role of the c subunit of the FOATP synthase in mitochondrial permeability transition," Cell Cycle, vol. 12, no. 4, pp. 674-683, 2013.

[8] N. García, N. Pavón, and E. Chávez, "The effect of N-ethylmaleimide on permeability transition as induced by carboxyatractyloside, agaric acid, and oleate," Cell Biochemistry and Biophysics, vol. 51, no. 2-3, pp. 81-87, 2008.

[9] P. S. Brookes, Y. Yoon, J. L. Robotham, M. W. Anders, and S.-S. Sheu, "Calcium, ATP, and ROS: a mitochondrial lovehate triangle," American Journal of Physiology-Cell Physiology, vol. 287, no. 4, pp. C817-C833, 2004.

[10] R. Chávez, N. Corona, C. García, and E. Chávez, "The effect of cyclosporin $\mathrm{A}$ on $\mathrm{Hg}^{2+}$-poisoning mitochondria. In vivo and in vitro studies," Comparative Biochemistry and Physiology Part C: Pharmacology, Toxicology and Endocrinology, vol. 107, no. 3, pp. 429-434, 1994.

[11] S. M. Korotkov, I. A. Skulskii, and V. V. Giazunov, "Cd" effects on respiration and swelling of rat liver mitochondria were modified by monovalent cations," Journal Inorganic Chemistry, vol. 556-562, 1998.

[12] E. A. Belyaeva, V. V. Glazunov, and S. M. Korotkov, "Cyclosporin A-sensitive permeability transition pore is involved in $\mathrm{Cd} 2+$-induced dysfunction of isolated rat liver mitochondria: doubts no more," Archives of Biochemistry and Biophysics, vol. 405, no. 2, pp. 252-264, 2002.

[13] N. Pavón, M. Buelna-Chontal, F. Correa et al., "Tamoxifen inhibits mitochondrial membrane damage caused by disulfiram," Biochemistry and Cell Biology, vol. 95, no. 5, pp. 556-562, 2017.

[14] R. F. Castillho, A. J. Kowaltowski, A. R. Meinicke, E. J. Bechara, and A. E. Vercesi, "Permeabilization of the inner mitochondrial membrane by $\mathrm{Ca}^{2+}$ ions is stimulated by t-butyl hydroperoxide and mediated by reactive oxygen species generated by mitochondria," Free Radic Biol Med, vol. 18, no. 3, pp. 479-486, 1995.

[15] M. M. Mahfouz, S. Q. Zhou, and F. A. Kummerow, "Vitamin $\mathrm{B}_{6}$ compounds are capable of reducing the superoxide radical and lipid peroxide levels induced by $\mathrm{H}_{2} \mathrm{O}_{2}$ in vascular endothelial cells in culture," International Journal for Vitamin and Nutrition Research, vol. 79, no. 4, pp. 218-229, 2009.

[16] L. Hernández-Esquivel, N. Pavón, M. Buelna-Chontal, H. González-Pacheco, J. Belmont, and E. Chávez, "Citicoline (CDP-choline) protects myocardium from ischemia/ reperfusion injury via inhibiting mitochondrial permeability transition," Life Sciences, vol. 96, no. 1-2, pp. 53-58, 2014.

[17] N. García, C. Zazueta, N. Pavón, and E. Chávez, "Agaric acid induces mitochondrial permeability transition through its interaction with the adenine nucleotide translocase. Its dependence on membrane fluidity," Mitochondrion, vol. 5, no. 4, pp. 272-281, 2005.

[18] O. H. Lowry, N. Rosebrough, A. L. Farr, and J. Randal, "Protein measurement with the folin phenol reagent," Journal of Biological Chemistry, vol. 193, pp. 262-275, 1951.

[19] I. Pérez-Torres, P. Roque, M. El Hafidi, E. Díaz-Díaz, and G. Baños, "Association of renal damage and oxidative stress in a rat model of metabolic syndrome. Influence of gender," Free Radical Research, vol. 43, no. 8, pp. 761-771, 2009.

[20] S. Dikalov, K. K. Griendling, and D. G. Harrison, "Measurement of reactive oxygen species in cardiovascular studies," Hypertension, vol. 49, no. 4, pp. 717-727, 2007.

[21] E. Majima, H. Oike, Y. M. Hong, Y. Shinohara, and H. Terada, "Characterization of cysteine residues of mitochondrial ADP/ ATP carrier with the SH-reagents eosin-5-maleimide and N-ethylmaleimide," Journal of Biological Chemistry, vol. 261, pp. 22181-22187, 1993.

[22] F. Correa, V. Soto, and C. Zazueta, "Mitochondrial permeability transition relevance for apoptotic triggering in the post-ischemic heart," The International Journal of Biochemistry \& Cell Biology, vol. 39, no. 4, pp. 787-798, 2007.

[23] B. Bataglia, A. Compagnone, A. Bandino et al., "Cobalt induces oxidative stress in isolated liver mitochondria responsible for permeability transition and intrinsic apoptosis in hepatocyte primary cultures," The International Journal of Biochemistry \& Cell Biology, vol. 41, pp. 586-594, 2009.

[24] A. Surguchev and A. Surguchov, "Effect of $\alpha$-synuclein on membrane permeability and synaptic transmission: a clue to neurodegeneration?" Journal of Neurochemistry, vol. 132, no. 6, pp. 619-621, 2015.

[25] S. Javadov, M. Karmazyn, and N. Escobales, "Mitochondrial permeability transition pore opening as a promising therapeutic target in cardiac diseases," Journal of Pharmacology and Experimental Therapeutics, vol. 330, no. 3, pp. 670-678, 2009.

[26] J. A. Hinson, D. W. Roberts, and L. P. James, "Mechanisms of acetaminophen-induced liver necrosis," Handbook of Experimental Pharmacology, vol. 196, pp. 369-405, 2010.

[27] A. Rasola and P. Bernardi, "Mitochondrial permeability transition in $\mathrm{Ca}^{2+}$-dependent apoptosis and necrosis," Cell Calcium, vol. 50, no. 3, pp. 222-233, 2011.

[28] P. Bernardi and P. Bonaldo, "Mitochondrial dysfunction and defective autophagy in the pathogenesis of collagen VI muscular dystrophies," Cold Spring Harbor Perspectives in Biology, vol. 5, no. 5, 2013.

[29] C. Zazueta, C. Sánchez, N. Garćia, and F. Correa, "Possible involvement of the adenine nucleotide translocase in the activation of the permeability transition pore induced by cadmium," The International Journal of Biochemistry \& Cell Biology, vol. 32, no. 10, pp. 1093-1101, 2000.

[30] Y. Wang, J. Fang, S. S. Leonard, and K. M. Krishna Rao, "Cadmium inhibits the electron transfer chain and induces reactive oxygen species," Free Radical Biology and Medicine, vol. 36, no. 11, pp. 1434-1443, 2004.

[31] E. N. Maciel, A. J. Kowaltowski, F. D. Schwalm et al., "Mitochondrial permeability transition in neuronal damage promoted by $\mathrm{Ca}^{2+}$ and respiratory chain complex II inhibition," Journal of Neurochemistry, vol. 90, no. 5, pp. 1025-1035, 2004. 
[32] T. Kano, E. E. Sato, S. Muranaka et al., "Oxidative stress underlies the mechanism for $\mathrm{Ca}^{(2+)}$-induced permeability transition of mitochondria," Free Radical Research, vol. 38, pp. 27-35, 2004.

[33] N. Tajeddine, "How do reactive oxygen species and calcium trigger mitochondrial membrane permeabilisation?" Biochimica et Biophysica Acta (BBA)-General Subjects, vol. 1860, no. 6, pp. 1079-1088, 2016.

[34] F. Treulen, P. Uribe, R. Boguen, and J. V. Villegas, "Mitochondrial permeability transition increases reactive oxygen species production and induces DNA fragmentation in human spermatozoa," Human Reproduction, vol. 30, no. 4, pp. 767-776, 2015.

[35] J. B. A. Custodio, A. J. M. Moreno, and K. B. Wallace, "Tamoxifen inhibits induction of the mitochondrial permeability transition by $\mathrm{Ca} 2+$ and inorganic phosphate," Toxicology and Applied Pharmacology, vol. 152, no. 1, pp. 10-17, 1998.

[36] Q. Chen, B. Gong, and A. Almasan, "Distinct stages of cytochrome $\mathrm{c}$ release from mitochondria: evidence for a feedback amplification loop linking caspase activation to mitochondrial dysfunction in genotoxic stress induced apoptosis," Cell Death \& Differentiation, vol. 7, no. 2, pp. 227-233, 2000.

[37] Q. Chen, Y.-C. Chai, S. Mazumder et al., "The late increase in intracellular free radical oxygen species during apoptosis is associated with cytochrome $\mathrm{c}$ release, caspase activation, and mitochondrial dysfunction," Cell Death \& Differentiation, vol. 10, no. 3, pp. 323-334, 2003.

[38] J. Suski, M. Lebiedzinska, M. Bonora, P. Pinton, J. Duszynski, and M. R. Wieckowski, "Relation between mitochondrial membrane potential and ROS formation," Mitochondrial Bioenergetics, vol. 1782, pp. 357-381, 2018.

[39] L. Hernández-Esquivel, N. Natalia-Pavón, C. Zazueta, N. García, F. Correa, and E. Chávez, "Protective action of tamoxifen on carboxyatractyloside-induced mitochondrial permeability transition," Life Sciences, vol. 88, no. 15-16, pp. 681-687, 2011. 